\title{
Tratamiento de las sibilancias recurrentes/asma en el niño menor de 3 años de edad
}

\author{
MI. Úbeda Sansano ${ }^{a}$, J. Murcia García ${ }^{b}$, JA. Castillo Laita ${ }^{c}$ \\ ${ }^{a}$ Pediatra. CS de La Eliana. Departamento 6. Valencia. \\ ${ }^{b}$ Pediatra. CS San Felipe. Jaén. \\ 'Pediatra. CS de Fuentes de Ebro. Zaragoza.
}

Rev Pediatr Aten Primaria. 2009; 1 1:97-120

M. ${ }^{\circ}$ Isabel Úbeda Sansano, ubeda_isa@gva.com

\section{Resumen}

Las guías y consensos actuales enfatizan la importancia del control del asma para disminuir la morbilidad y mejorar la calidad de vida de estos pacientes. En niños menores de 3 años, conseguir un adecuado control resulta más difícil que a otras edades debido a la heterogeneidad de fenotipos de sibilantes recurrentes, patrón inflamatorio predominante y factores desencadenantes, probablemente distintos y evolución y respuesta al tratamiento variables. Identificar el fenotipo sibilante podría ayudar a tomar decisiones terapéuticas, aunque en ocasiones se solapan en un mismo paciente características de distintos fenotipos y el tratamiento debería individualizarse en función de la respuesta.

Se revisan los tres pilares básicos del tratamiento: educación, medidas preventivas y tratamiento farmacológico tanto del episodio agudo como de mantenimiento. Los fármacos recomendados para el tratamiento de mantenimiento en menores de 3 años son los corticoides inhalados (CI) y los inhibidores de leucotrienos. Los Cl son de elección en el asma y la respuesta es más satisfactoria ante niños con sibilancias y atopia. No existen evidencias para recomendar dosis bajas de $\mathrm{Cl}$ en la prevención de episodios de sibilancias desencadenadas exclusivamente por virus a esta edad; los inhibidores de los leucotrienos estarían más indicados en estos casos. También constituyen una alternativa a los $\mathrm{Cl}$ en el asma leve y podrían asociarse a ellos para disminuir las exacerbaciones inducidas por virus. No obstante, algunos niños no responden a ninguno de estos fármacos. Se recomienda revisar periódicamente el tratamiento y suspenderlo o considerar un diagnóstico o tratamiento alternativo si no se observan beneficios.

Palabras clave: Sibilancias recurrentes, Asma, Tratamiento, Niños, Preescolares, Lactantes.

Abstract

Today's guides and consensus emphasize the importance of asthma control in diminishing morbidity and improving these patients' quality of life. This control is not easy in children under 3 years of age due to: recurrent wheezing phenotype heterogeneity, triggering factors and predominant inflammatory pattern (probably different), and variable evolution and treatment response.

Los autores declaran no presentar conflictos de intereses en relación con la preparación y publicación de este artículo. 
Identifying the wheezing phenotype could help making therapeutic decisions. However, characteristics from different phenotypes can sometimes overlap in a patient, so the treatment should be adjusted according to the patient's response.

We review the 3 basic pillars of treatment: education, preventive measures and drug treatment. We do this for the acute episode and for the treatment maintenance.

The recommended drugs on children under 3 are inhaled corticoids (IC) and leukotriene inhibitors. IC are the treatment of choice for asthma and there is a better response on children with wheezing plus atopy. There is no evidence in the recommendation of low IC doses in the prevention of wheezing episodes exclusively triggered by virus on children of this age. Leukotriene inhibitors are more appropriate in these cases. They are an alternative to IC on children with mild asthma and they could be used to decrease exacerbations induced by virus.

Some children, however, do not respond to any of these drugs. It is recommended to review the treatment periodically and suspend it or consider an alternative treatment or diagnosis if benefits are not observed.

Key words: Recurrent wheezing, Asthma, Treatment, Children, Preschool, Infant.

\section{Introducción}

Las guías y consensos actuales enfatizan la importancia del control del asma, pero a pesar de los conocimientos científicos y del amplio arsenal terapéutico disponible, este control no es fácil en el grupo de niños de corta edad en los que las sibilancias recurrentes, tos y disnea, manifestaciones típicas del asma, siguen causando elevada morbilidad y gran consumo de recursos.

En este artículo se intentará dar respuesta a aquellas preguntas que nos hacemos en la práctica clínica a la hora de plantear el tratamiento de las sibilancias recurrentes en un niño menor de 3 años de edad. Debido a las dificultades, su diagnóstico y tratamiento suponen un verdadero reto para los pediatras.

\section{¿A qué se deben las dificultades terapéuticas?}

El primer problema ante el que nos encontramos es establecer previamente el diagnóstico de certeza de asma en un niño menor de 3 años. Según el Consenso Internacional Pediátrico de Asma ${ }^{1}$, en los niños pequeños en los que resulta difícil efectuar pruebas funcionales, el diagnóstico se realiza ante el antecedente de 3 o más episodios de sibilancias y/o tos persistentes, en una situación en la que el asma es probable y se han descartado otras enfermedades menos frecuentes. A pesar de que esta definición sigue vigente en consensos actuales de asma ${ }^{2,3}$, en la práctica clínica su aplicación no es tan sencilla como parece ${ }^{4}$. Es precisamente en estas edades donde más difícil resulta establecer el diagnóstico de asma con seguridad, ya que cuan- 
to menor es la edad del niño, mayor es la probabilidad de encontrarnos con otras entidades menos frecuentes (malformaciones cardiopulmonares, fibrosis quísti$\mathrm{ca}$, displasia broncopulmonar, aspiración de cuerpo extraño, reflujo gastroesofágico, etc.). Además, aun descartando estas patologías, se ha visto por estudios longitudinales de cohortes que la evolución de los niños pequeños con sospecha de asma es muy heterogénea. De ahí que a veces se prefiera, en muchos de estos lactantes y preescolares, utilizar el término de sibilancias al de asma, como apunta la reciente publicación de la European Respiratory Society (ERS) Task Force ${ }^{5}$.

En el estudio de Melbourne ${ }^{6}$, hasta el $60 \%$ de los niños que se etiquetaron de "bronquitis silbante" en los primeros 7 años de vida, se encontraban asintomáticos a los 42 años, mientras que en el $70 y$ 90\% de los que a esa misma edad diagnosticaron de asma y asma grave respectivamente, los síntomas persistían en la edad adulta. La sensibilización precoz a alérgenos también supuso mayor riesgo y deterioro de la función pulmonar. Ello hace pensar que los niños sensibilizados a algún alérgeno o con síntomas más graves, tienen mayor probabilidad de padecer asma.

En la cohorte de Tucson ${ }^{7}$, donde se han seguido de forma periódica durante más de 20 años a 1.246 recién nacidos entre 1980 y 1984, también se han observado distintos fenotipos o patrones de sibilancias recurrentes en la infancia, que difieren en características, evolución y respuesta al tratamiento ${ }^{8}$ :

- Sibilancias precoces transitorias: los síntomas generalmente comienzan en el primer año de vida y se resuelven alrededor de los 3 años. Representan el $40-60 \%$ de las sibilancias recurrentes en menores de 3 años. No suelen asociar historia familiar de asma o sensibilización a alérgenos y el principal factor de riesgo es la disminución de la función pulmonar al nacer. También se encontró asociación con la prematuridad, tabaquismo pasivo pre- y posnatal, ser varón, tener hermanos mayores y asistencia a guardería.

- Sibilancias persistentes no atópicas: suelen comenzar también en el primer año de vida, generalmente tras una bronquiolitis y persisten hasta el comienzo de la adolescencia. La hiperreactividad bronquial va disminuyendo con la edad. Suponen alrededor del 20\% de las sibilancias a esta edad. Tampoco suelen asociar historia personal o familiar de atopia y la función pulmonar es normal al nacimiento, 
aunque se encuentra disminuida en la adolescencia.

- Sibilancias persistentes atópicas: habitualmente tienen su primer episodio después del primer año de vida y persisten en la adolescencia y edad adulta. Representan alrededor del $20 \%$ de las sibilancias en menores de 3 años. La función pulmonar es normal al nacimiento pero disminuye en los primeros años de vida. Se asocia con hiperreactividad bronquial e historia familiar y personal de atopia y suele predominar en varones. Del estudio de Tucson ${ }^{8}$ se concluye que hasta en el $80 \%$ de los lactantes y preescolares, las sibilancias son desencadenadas fundamentalmente por infecciones víricas, sin relación con la atopia y la clínica es transitoria. Ello justificaría la escasa respuesta terapéutica que muchos de estos niños tienen al tratamiento convencional del asma'. En los sibilantes persistentes, la característica fundamental es la presencia de atopia y sensibilización precoz a alérgenos, pero ello no es un obstáculo para que las infecciones víricas se comporten también como desencadenantes en estos niños.

Recientemente la ERS ha clasificado las sibilancias de los preescolares en función de su duración (transitorias, persistentes, de comienzo tardío) y del patrón temporal de estas (episódicas desencadenadas solo por virus o las producidas por múltiples desencadenantes) 5 .

Aunque en principio podríamos pensar que la identificación del tipo de sibilante nos ayudará a tomar decisiones, la realidad no siempre es tan sencilla, ya que en ocasiones se solapan en un mismo paciente características de distintos fenotipos. Probablemente se deba, como apuntan las nuevas líneas de investigación, a que existen además otros factores, entre ellos genéticos, que condicionarían la respuesta terapéutica no solo en función del fenotipo.

\section{Patrón de inflamación: ¿es similar en todos los niños menores de 3 años?}

El dato histológico que caracteriza al asma es la inflamación crónica de la vía aérea en la que intervienen distintas células y mediadores $\mathrm{y}$, aunque clásicamente se ha asociado con eosinofilia, este no es el único patrón inflamatorio observado en la evolución de la enfermedad. En el lavado broncoalveolar y biopsia de lactantes con sibilancias recurrentes y limitación reversible al flujo aéreo, incluso en presencia de atopia, se observó un predominio neutrofílico sin cambios estructurales (remodelación) en la vía aérea ${ }^{10}$. Sin embargo, a los 3 
años, los niños que tuvieron antecedentes de sibilancias y mayor gravedad de la enfermedad, mostraban ya un predominio de eosinófilos y cambios anatomopatológicos de remodelación ${ }^{11}$. La respuesta al tratamiento parece ser distinta en función del predominio de células inflamatorias (neutrófilos o eosinófilos) en el árbol bronquial.

\section{Índice predictivo de asma}

Con la intención de facilitar el diagnóstico de asma, ante la ausencia de marcadores biológicos sensibles y específicos y la limitación de otras pruebas diagnósticas, se describió el índice predictivo de asma (IPA) de Castro-Rodríguez y cols. ${ }^{12}$ o modificaciones de este ${ }^{13}$ que, pese a sus limitaciones, es una herramienta sencilla, aplicable a niños menores de 3 años con sibilancias recurrentes y puede resultar útil en la toma de decisiones (tabla I). El IPA se considera positivo (riesgo de padecer asma) si existe al menos un criterio mayor o dos menores. Según Castro Rodríguez, un niño menor de 3 años con sibilancias recurrentes e IPA positivo tiene una probabilidad del $77 \%$ de padecer asma atópica en la edad escolar (6-13 años), VPP (valor predictivo positivo) $=76,6 \%$ (IC [intervalo de confianza] 95\%: 73,479,8); si el IPA es negativo, la probabilidad de no tener asma atópica a esa edad es de $68 \%$, VPN (valor predictivo negativo) $=68,3 \%$ (IC 95\%: 64,771,9). Los niños con IPA positivo tienen 7 veces más riesgo de tener asma en la edad escolar que los IPA negativo (OR [odds ratio] = 7,1; IC 95\%: 3,5-14,1).

En resumen, la historia cuidadosa sobre los antecedentes del niño y su familia, la presencia o no de otros signos o síntomas acompañantes, forma de pre-

Tabla I. Índice predictivo de asma

\begin{tabular}{|c|c|}
\hline Castro Rodríguez ${ }^{12}$ & Guilbert ${ }^{13}$ \\
\hline \multicolumn{2}{|c|}{ Criterios mayores } \\
\hline Historia de asma en algún padre & Historia de asma en algún padre \\
\hline $\begin{array}{l}\text { Diagnóstico médico de dermatitis } \\
\text { atópica en el niño }\end{array}$ & $\begin{array}{c}\text { Diagnóstico médico de dermatitis } \\
\text { atópica en el niño } \\
\text { Sensibilización alérgica a } \\
\text { uno o más aeroalérgenos }\end{array}$ \\
\hline \multicolumn{2}{|c|}{ Criterios menores } \\
\hline $\begin{array}{l}\text { Rinitis alérgica diagnosticada } \\
\text { por un médico }\end{array}$ & $\begin{array}{c}\text { Sensibilización alérgica a huevo, } \\
\text { leche o frutos secos }\end{array}$ \\
\hline Sibilancias no relacionadas con resfriados & Sibilancias no relacionadas con resfriados \\
\hline Eosinofilia en sangre periférica $\geq 4 \%$ & Eosinofilia en sangre periférica $\geq 4 \%$ \\
\hline
\end{tabular}


sentación y edad de inicio de las sibilancias, coexistencia con infecciones, su duración, la existencia o no de periodos asintomáticos, la sensibilización a alérgenos, la respuesta a tratamientos previos, junto con la exploración del paciente, orientarán el diagnóstico y la pauta a seguir (figura 1$)^{9}$. Se sabe que la sensibilización al huevo en el primer año de vida es un marcador de sensibilización y manifestaciones de alergia a neumoalérgenos a los 7 años de edad.

\section{Tratamiento}

Se revisa el tratamiento siguiendo las recomendaciones actuales para el asma, pero teniendo en cuenta las peculiaridades que presentan los niños a esta edad.

Si consideramos las dificultades diagnósticas en este rango de edad, la heterogeneidad de los fenotipos, patrón inflamatorio predominante y factores desencadenantes probablemente distintos y la respuesta al tratamiento y evolución variables, es fácil entender que, a diferencia de lo que ocurre a otras edades, existan más incertidumbres de cómo actuar ante estos niños. Así, y a pesar de que últimamente se están haciendo esfuerzos para diferenciar el tratamiento en este grupo de edad, todavía existen muchas incógnitas por

Figura 1. Algoritmo de actuación ante sospecha de asma en preescolares.

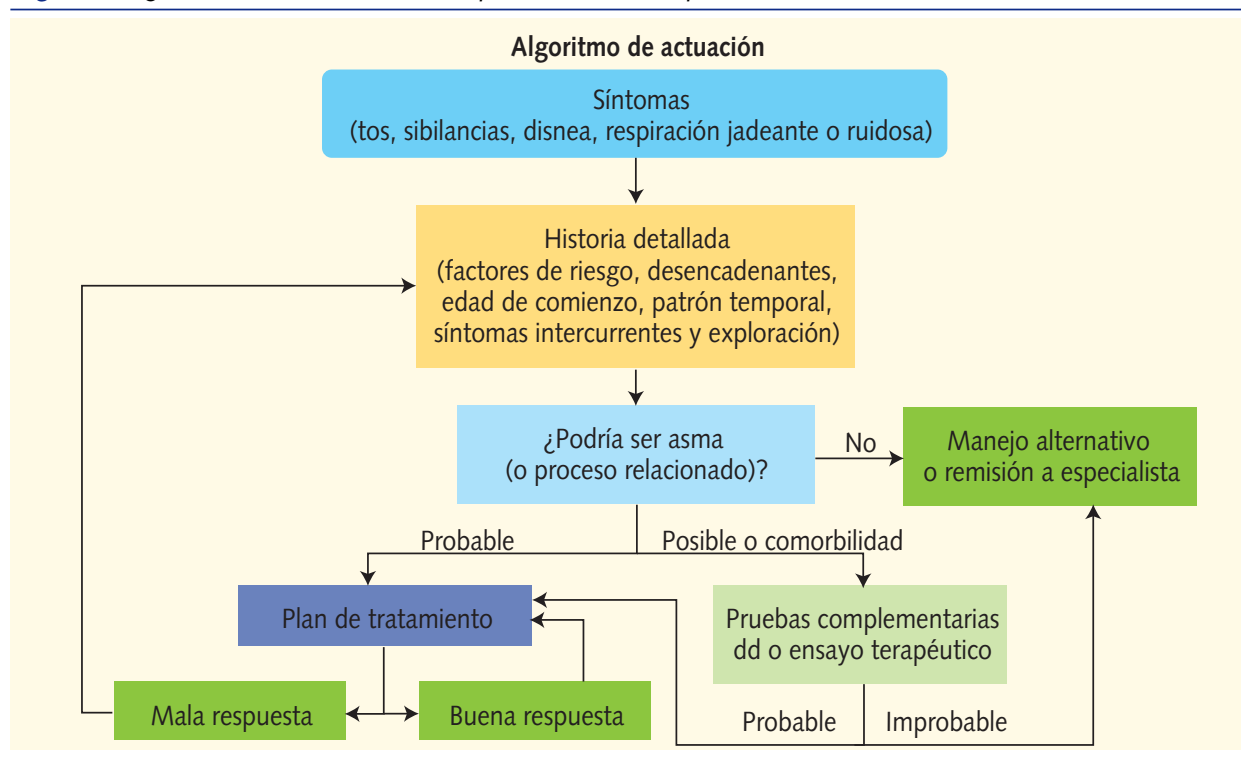

dd: diagnóstico diferencial. 
resolver y la evidencia científica que lo sustenta es menor que en los niños mayores, por lo que el tratamiento deberá individualizarse en cada caso.

Igual que en pacientes de mayor edad, la educación, medidas preventivas y el tratamiento farmacológico son tres pilares básicos del tratamiento en los niños menores de 3 años.

\section{Educación}

La información y educación son esenciales en el cuidado de estos niños.

Antes de tomar cualquier decisión, es fundamental que los padres o cuidadores conozcan la naturaleza del problema diagnóstico y terapéutico a estas edades. Debemos exponerles nuestras incertidumbres o sospechas para que no tengan falsas expectativas sobre la efectividad del tratamiento, ya que la respuesta no siempre es satisfactoria; ni infundirles una preocupación indebida, ya que no en todos los niños van a persistir los síntomas.

En el asma se ha demostrado que los programas educativos encaminados a reconocer los síntomas y el control en el domicilio mediante planes escritos, junto con la información sobre la enfermedad y la utilidad de los distintos tratamientos, adquisición de habilidades para administrar el tratamiento y las re- visiones médicas periódicas, no solo mejora el control de la enfermedad y la calidad de vida del paciente, sino que resulta una medida coste-efectiva ${ }^{14}$. Por las características de comportamiento de los niños pequeños, a veces a los padres les resulta difícil administrar la medicación inhalada, por ello es muy importante adiestrar a los cuidadores y comprobar que la técnica de administración es correcta con el dispositivo adecuado a la edad, ya que independientemente de si precisan o no medicación de mantenimiento por vía inhalada, en los episodios agudos, el uso de la vía inhalatoria es de elección para administrar el tratamiento farmacológico.

La educación debe realizarse de forma progresiva, secuencial, organizada y estructurada dentro de un programa, pero sabiendo que cualquier ocasión puede ser útil para llevar a cabo o reforzar alguna acción educativa. Para ello es imprescindible motivar y formar tanto a los profesionales sanitarios que atienden al niño (médicos, enfermería) como a los familiares o cuidadores y profesores.

\section{Medidas preventivas}

- Alimentación. La lactancia materna ejerce un efecto protector sobre la aparición precoz de sibilan- 
cias $^{15}$, que parece mayor en niños con riesgo de atopia y cuando la lactancia se prolonga al menos 4 meses. Aunque no en todos los estudios se han observado estas ventajas, el potencial efecto protector sobre la aparición precoz de asma y otros beneficios bien conocidos de la lactancia materna, justifican que sea el alimento de elección en los primeros 6 meses de vida, como recomienda la OMS, e iniciar posteriormente la alimentación complementaria.

La introducción tardía de alimentos sólidos (por encima de los 6 meses de edad) no constituye un factor protector para disminuir la sensibilización a alérgenos alimentarios o neumoalérgenos, ni previene la aparición de asma o rinitis alérgica ${ }^{16}$.

Revisiones sistemáticas de la Colaboración Cochrane han demostrado que en niños de riesgo, las restricciones dietéticas maternas durante el embarazo y la lactancia y la sustitución, en la alimentación del niño, de proteínas de vaca por leche de soja, no retrasan la aparición de alergias. Tampoco existe evidencia en este sentido para recomendar hidrolizados de pro- teínas vacunas, prebióticos o probióticos.

- Vacuna antigripal. No está claro el beneficio de la vacuna antigripal en la prevención de las exacerbaciones de asma, pero sí parece improbable, como se ha demostrado por recientes ensayos clínicos, que esta empeore el asma, al menos tras la administración de la vacuna de virus inactivados, que es la única recomendada en la infancia disponible en España. Mayores precauciones se deberían tomar en niños asmáticos con la nueva vacuna intranasal de virus vivos atenuados, que aunque parece más efectiva que la anterior en la prevención de la gripe, se han observado mayor tasa de sibilancias e ingresos hospitalarios en lactantes ${ }^{17}$.

- Tabaco. El hábito tabáquico materno durante el embarazo es un factor de riesgo para desarrollar episodios recurrentes de sibilancias durante los dos primeros años de vida $(O R=2,2 ;$ IC 95\%: 1,3$3,6)^{18,19}$. Ya se ha descrito previamente que los preescolares con el fenotipo de sibilancias precoces transitorias tienen una disminución de la función pulmonar al 
nacer y mayor riesgo de hiperreactividad bronquial en el periodo de lactante, antes de haber tenido el primer episodio de sibilancias. Uno de los factores de riesgo prevenibles de este fenotipo es el tabaquismo pasivo durante el embarazo, por tanto, es importante fomentar el abandono de este hábito en las mujeres gestantes.

La exposición a un ambiente con humo de tabaco también es un factor de riesgo para padecer episodios recurrentes de sibilancias en la infancia precoz. El niño fumador pasivo tiene más riesgo de infecciones respiratorias de vías altas y agravamiento del asma ${ }^{20}$. De hecho, algunos autores han incluido el tabaquismo familiar, sobre todo materno, como un criterio mayor a considerar en el IPA ${ }^{21}$.

Se debe informar a las gestantes sobre la conveniencia de abandonar el hábito tabáquico durante el embarazo y recomendar que el niño/a viva en un ambiente libre de humo.

\section{Tratamiento farmacológico}

Se describen los fármacos indicados para el tratamiento del episodio agudo y el control de la enfermedad.

\section{Tratamiento del episodio agudo}

La crisis o agudización se define como un episodio de inicio brusco o gradual de tos, disnea, sibilancias, disminución de la tolerancia al ejercicio, trastornos de la alimentación o trastornos del sue-

\begin{tabular}{|c|c|c|c|c|}
\hline \multirow[t]{2}{*}{ Puntuación } & \multicolumn{2}{|c|}{$\begin{array}{l}\text { Frecuencia } \\
\text { respiratoria }\end{array}$} & \multirow[t]{2}{*}{ Sibilancias } & \multirow{2}{*}{$\begin{array}{c}\text { Uso de músculos } \\
\text { accesorios } \\
\text { (esternocleidomastoideo) }\end{array}$} \\
\hline & $<6$ años & $\geq 6$ años & & \\
\hline 0 & $<30$ & $<20$ & No & No \\
\hline 1 & $31-45$ & $21-35$ & $\begin{array}{c}\text { Final espiración } \\
\text { (estetoscopio) }\end{array}$ & Incremento leve \\
\hline 2 & $46-60$ & $36-50$ & $\begin{array}{c}\text { Toda la espiración } \\
\text { (estetoscopio) }\end{array}$ & Aumentado \\
\hline 3 & $>60$ & $>50$ & $\begin{array}{c}\text { Inspiración y espiración, } \\
\text { sin estetoscopio*** }\end{array}$ & Actividad máxima \\
\hline \multicolumn{5}{|c|}{$\begin{array}{l}\text { El uso de músculos accesorios se refiere solo al esternocleidomastoideo, que es el único músculo que se ha corre- } \\
\text { lacionado bien con el grado de obstrucción. } \\
\text { * Se puntúa de } 0 \text { a } 3 \text { en cada uno de los apartados (mínimo 0, máximo 9). } \\
\text { * * Si no hay sibilancias y la actividad del esternocleidomastoideo está aumentada, puntuar el apartado sibilan- } \\
\text { cias con un } 3 .\end{array}$} \\
\hline
\end{tabular}


ño, reversibles espontáneamente o con fármacos broncodilatadores.

En los niños menores de 3 años, el tratamiento de la crisis se fundamenta en la extrapolación de datos de la experiencia clínica-investigadora del asma en pacientes más mayores, pues existen escasos estudios y protocolos referidos a esta edad ${ }^{2,22}$.

Antes del abordaje terapéutico es fundamental valorar la gravedad de la crisis.

\section{Valoración de la gravedad}

La gravedad de la crisis se valora en base a la clínica (frecuencia respiratoria, presencia de sibilancias y uso de los músculos esternocleidomastoideos), variables recogidas en el pulmonary score (PS) (tabla II)23. Esta escala tiene como ventajas su sencillez y aplicabilidad en todas las edades. Junto a la pulsioximetría $\left(\mathrm{SpO}_{2}\right)$, permite valorar mejor la crisis, sobre todo en niños pequeños en los que la gravedad se correlaciona menos con la clínica (tabla III)².
Es fundamental que los centros de salud dispongan de pulsioxímetro para conocer la gravedad de la crisis asmática.

Fármacos y vías de administración. ¿Responden todos los niños?

- $\beta_{2}$-agonistas de acción rápida. Son el tratamiento broncodilatador de primera línea en las crisis asmáticas y muy empleados para tratar las sibilancias en lactantes y preescolares. No existe evidencia de qué broncodilatador es más eficaz para los distintos tipos de sibilantes y cuál es la dosis óptima. En los menores de 2 años, los resultados de un metaanálisis de la revisión Cochrane muestran datos contradictorios, unos niños responden y otros no. Se concluye que no existen beneficios claros de su utilización en las sibilancias recurrentes a esta edad ${ }^{24}$. Parece que la eficacia es mayor en aquellos niños con factores de riesgo

Tabla III. Valoración global de la gravedad de la crisis integrando el pulmonary score y la saturación de oxígeno

\begin{tabular}{lcc}
\hline & $\mathrm{PS}$ & $\mathrm{SpO}_{2}$ \\
\hline Leve & $0-3$ & $>94 \%$ \\
Moderada & $4-6$ & $91-94 \%$ \\
Grave & $7-9$ & $<91 \%$ \\
\hline
\end{tabular}

En caso de discordancia entre la puntuación clínica y la saturación de oxígeno se utilizará el de mayor gravedad. PS: pulmonary score; $\mathrm{SpO}_{2}$ : saturación de oxígeno. 
para desarrollar asma atópica. No obstante, debido a la respuesta individual tanto en lactantes como en preescolares y a que es difícil saber quién va a responder, está justificada una prueba terapéutica y observar su efecto.

Por su mayor efectividad y menos efectos secundarios, la vía inhalatoria es la de elección para administrar el tratamiento.

El uso del inhalador presurizado con cámara espaciadora es tan efectiva (o más) como los nebulizadores en el tratamiento del episodio agudo de asma $^{2}$ y origina menos efectos secundarios (taquicardia, hipoxia) en los niños ${ }^{25}$. En el caso de crisis graves, donde además se debe administrar siempre oxígeno, sería de elección el tratamiento nebulizado. En el asma de riesgo vital, las crisis deben tratarse siempre con broncodilatador nebulizado.

Se recomienda salbutamol a una dosis que depende de la gravedad de la crisis y de la respuesta inicial, en tandas de 2-10 pulsaciones de $100 \mu \mathrm{g}$ hasta conseguir una respuesta. En las crisis leves puede ser suficiente una tanda de 2-4 pulsaciones $^{2}$. El salbutamol nebulizado se administrará a dosis de 0,15 $\mathrm{mg} / \mathrm{kg}$, máximo $5 \mathrm{mg}(1 \mathrm{ml})$, disuelto en 2-3 $\mathrm{ml}$ de suero fisiológico. Cuando se pauta con oxígeno, el flujo debe ser elevado, nunca inferior a 6-9 litros/minuto.

La equivalencia de dosis entre sistemas de inhalación no está aclarada, ya que aunque la dosis nebulizada recomendada es mucho más elevada, el depósito pulmonar del fármaco también es menor. No obstante, hay que tener en cuenta que la dosis mínima nebulizada $(0,25$ $\mathrm{ml}$ ) equivale a 12 pulsaciones de salbutamol, lo que probablemente justifique los mayores efectos adversos observados. Benito y cols. ${ }^{26}$ recomiendan reconsiderar la indicación de dosis altas en la mayoría de las crisis asmáticas, pues no encuentra diferencias significativas en la respuesta a distintas dosis.

- Bromuro de ipratropio. Es otro broncodilatador que se ha usado con frecuencia ante sibilancias recurrentes en niños pequeños, la mayoría desencadenadas por infecciones víricas, pero no hay evidencias para emplearlo en monoterapia, incluso en los menores de 2 años, en los que se pensaba que pudieran ser más útiles ${ }^{27}$. 
Su empleo sólo está justificado en el tratamiento de las crisis moderadas o graves y siempre asociado a los $\beta_{2}$-agonistas de acción corta. No se recomienda su uso de forma sistemática en todos los episodios agudos, el beneficio es mayor en el niño con asma grave².

Aunque no se conoce con exactitud cuál es la dosis óptima, en menores de $30 \mathrm{~kg}$ se recomiendan $250 \mu \mathrm{g} / 4-6$ horas si se administra nebulizado y 40-80 $\mu \mathrm{g}$ (2-4 pulsaciones) si se hace con inhalador presurizado y cámara. El efecto máximo, que no se mantiene, se da con las primeras dosis, por lo que solo se recomienda en las primeras 24-48 horas². No se ha mostrado útil en la disminución de la estancia en niños hospitalizados.

- Corticoides. Numerosos estudios han demostrado que cuando se usan de forma precoz en una crisis, reducen significativamente la necesidad de hospitalizaciones, las recaídas y el uso de $\beta_{2}$-agonistas. Están indicados, asociados a los $\beta_{2}$-agonistas de acción corta, en todas las crisis moderadas o graves, en niños con antecedentes de crisis graves y en los episodios leves cuando la mejoría con los broncodilatadores no persiste al menos durante 4 horas $^{2}$.

La dosis recomendada es de 0,5-1 $\mathrm{mg} / \mathrm{kg}$ de prednisona o equivalente en crisis leves-moderadas, o 1-2 $\mathrm{mg} / \mathrm{kg} /$ día en crisis graves (máximo 50-60 mg) durante 3-10 días o hasta la resolución. Se puede suspender sin reducción de la dosis si se mantiene menos de 10 días. Salvo imposibilidad de utilizarla, la vía oral es la de elección para administrar los corticoides en los episodios agudos, su eficacia es similar a la parenteral. No hay suficiente evidencia para recomendar corticoides inhalados a altas dosis (2,4 mg de budesonida o 2 $\mathrm{mg}$ de fluticasona), equivalentes al uso de corticoides orales, además de que resulta una práctica cara. Tampoco se recomienda, en el episodio agudo, duplicar las dosis de corticoides inhalados si los Ilevaba pautados previamente.

No han encontrado diferencias, en términos de eficacia, entre la utilización de los diversos corticoides. No existen suficientes datos para recomendar el tratamiento con corticoides orales a criterio de los padres en las reagudizaciones. Las pruebas al respecto son limitadas 
para establecer conclusiones clíni-

cas y no se puede hacer un uso generalizado de esta estrategia hasta que se aclaren en profundidad los posibles daños y beneficios. No obstante, padres bien en-

Figura 2. Tratamiento de las crisis asmática.

\section{Crisis leve}

$\beta_{2}$-agonista inhalado: 2-4 pulsaciones en cámara ${ }^{a}$

(Cada 20 minutos hasta 3 tandas)

$\beta_{2}$-agonista nebulizado ${ }^{b}$

(1 dosis: $0,15 \mathrm{mg} / \mathrm{kg}$-salbutamol-)

Valorar corticoides orales si los recibía previamente

Reevaluar en 15 minutos

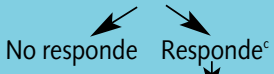

$y$

Alta al domicilio

$\beta_{2}$-agonista a demanda

Corticoides si se iniciaron

Reevaluación en 2-3 días

\section{Crisis moderada}

$\beta_{2}$-agonista inhalado: 6-8 pulsaciones en cámara ${ }^{a}$

(Cada 20 minutos hasta 3 tandas)

$$
0
$$

$\beta_{2}$-agonista nebulizado ${ }^{b}$

$(0,15 \mathrm{mg} / \mathrm{kg}$-salbutamol-. Cada 20 minutos hasta 3 tandas)

$+$

Valorar bromuro de ipratropio inhalado ( 2 a 4 pulsaciones) o nebulizado Una tanda cada 20 minutos. Máximo 3 tandas

$$
+
$$

Corticoides orales

$$
+
$$

Oxígeno hasta $\mathrm{SpO}_{2}>94 \%$

$$
\downarrow
$$

Reevaluar en 15 minutos tras última dosis

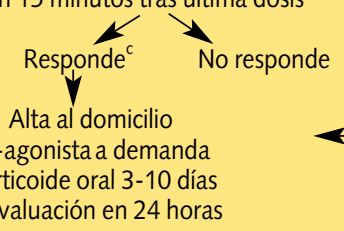

\section{Crisis grave}

Oxígeno hasta $\mathrm{SpO}_{2}>94 \%$ $+$

$\beta_{2}$-agonista inhalado: 8-10 pulsaciones en cámara ${ }^{a}$ (Cada 20 minutos hasta 3 tandas)

$$
0
$$

$\beta_{2}$-agonista nebulizado

$(0,15 \mathrm{mg} / \mathrm{kg}$-salbutamol-. Cada 20 minutos hasta 3 tandas)

$$
+
$$

Bromuro de ipratropio

inhalado (2 a 4 pulsaciones) o nebulizado

Una tanda cada 20 minutos. Hasta 3 tandas

Corticoides orales (2 mg/kg) oral/IV

$\downarrow$

Reevaluar en 15 minutos tras última dosis

\section{${ }^{a}$ De elección.}

${ }^{b}$ Mínimo 1,25 mg $(0,25 \mathrm{ml})$, máximo $5 \mathrm{mg}(1 \mathrm{ml})$.

${ }^{c}$ Responde si pulmonary score $<4$ y $\mathrm{SpO}_{2}$ (saturación de oxígeno) $>94 \%$. 
trenados en el automanejo podrían iniciar el tratamiento domiciliario en los casos necesarios sin mayor riesgo ${ }^{28}$. La administración de ciclos cortos de corticoides orales repetidos (3 ciclos en el último año) no se asocia con alteraciones del metabolismo óseo, de la densidad mineral ósea o de la función adrenal ${ }^{29}$.

\section{Manejo del episodio agudo}

Cualquier tipo de crisis asmática o episodio de sibilancias recurrentes puede ser tratado inicialmente en Atención Primaria.

En la figura 2 se expone el esquema de actuación ante una crisis de as$\mathrm{ma}^{2,30,31}$, que en principio podríamos extrapolar para un episodio agudo de un lactante o preescolar con sibilancias recurrentes.

\section{Ante un episodio agudo debemos recordar}

- Es importante revisar siempre la técnica de inhalación (muchos fracasos terapéuticos se deben a una mala técnica).

- Los broncodilatadores $\beta_{2}$-agonistas de acción corta son los fármacos de elección en todo tipo de crisis y aunque no todos los niños menores de 3 años responden al tratamiento, está justificado hacer un ensayo terapéutico y valorar la respuesta. Se administrarán a demanda, utilizando inhalador presurizado con cámara espaciadora y mascarilla facial. El tratamiento nebulizado debe reservarse para situaciones graves, utilizando en estos casos oxígeno a flujo alto y no aire comprimido.

- Los corticoides sistémicos se recomiendan en todas las crisis moderadas y graves. Se administrarán lo antes posible (en la primera hora desde inicio del tratamiento).

- Se debe administrar oxígeno en todos los pacientes con $\mathrm{SpO}_{2}<$ 94\% (si tras la administración del broncodilatador permanece la situación de hipoxia). Si no disponemos de pulsioxímetro se administrará siempre en crisis moderadas y graves. Se administrará a flujo alto (6-9 litros/minuto) y continuo mediante mascarillas o sondas nasales.

- Se deberá entregar un plan escrito del tratamiento a seguir antes de remitir el niño a su domicilio y recomendar seguimiento estrecho en las siguientes 24-48 horas por su pediatra.

- En caso de no mejorar el paciente, 
o si la duración de la mejoría es menor de 3 horas, se tratará como una crisis grave, valorando la derivación al hospital más próximo en ambulancia medicalizada acompañado por personal sanitario y administrando durante el transporte oxígeno, broncodilatadores y corticoides.

- No se recomienda el uso de mucolíticos, antihistamínicos, fisioterapia torácica ni antibióticos (la mayoría de las crisis son desencadenadas por infecciones víricas).

\section{Tratamiento farmacológico de mantenimiento}

La decisión de cuándo iniciar un tratamiento diario resulta difícil en estos pacientes $^{32}$. Dependerá de qué niño preescolar con sibilancias recurrentes estemos controlando para dar respuesta a cuestiones tales como si precisa o no tratamiento de mantenimiento, cuál es el más indicado y si responderá al mismo. Siguiendo las recomendaciones del consenso español sobre el tratamiento del asma en Pediatría ${ }^{2}$ se establecen las pautas terapéuticas para menores de 3 años (tabla IV).

Se ha discutido que, en este rango de edad, si pautamos un tratamiento antiinflamatorio específico para el asma, es posible que estemos tratando como asmáticos a muchos niños sin serlo, pero, por contra, también se puede caer en el error de considerar todos los casos como transitorios y no plantear un tratamiento apropiado ante el temor de estigmatizar con una enfermedad crónica a estos pacientes. No olvidemos que en el $80 \%$ de los asmáticos, las sibilancias están ya presentes antes de los 6 años y la inflamación y cambios estructurales en el bronquio aparecen de forma precoz.

El tratamiento de mantenimiento se iniciará en función de la recurrencia o intensidad de los síntomas y sospecha del tipo de sibilante, pero ante todo debe prevalecer el juicio clínico o sentido común, sabiendo que no en todos estos niños vamos a encontrar la respuesta deseada. Una actitud prudente ante un paciente con síntomas frecuentes o persistentes, es comenzar de forma escalonada siguiendo las recomendaciones de las guías y consensos de asma e identificar la respuesta individual de cada paciente. Si la respuesta es satisfactoria, se mantendrá la misma pauta, al menos durante 3 meses, hasta considerar descender un escalón o evaluar la necesidad de mantener un tratamiento. Si en el plazo de 4-6 semanas no se observa ningún beneficio tras haber comproba- 
do que la administración y cumplimentación son correctas, se debería suspender el tratamiento o buscar otras alternativas diagnósticas o terapéuticas. La mayoría de los casos pueden controlarse en las consultas de Atención Primaria, pero debemos valorar la indicación de derivar a una consulta especializada en función de la gravedad de los síntomas o si existen dudas diagnósticas.

Los únicos fármacos disponibles que han demostrado su utilidad en el control del asma a esta edad son los corticoides inhalados $(\mathrm{Cl})$ y los inhibidores de los leucotrienos. No existen datos para avalar la indicación de los $\beta_{2}$-agonistas de acción prolongada asociados a los corticoides en estos casos y las cromonas no parecen mejor que placebo. Aunque se ha descrito el efecto antiinflamatorio de los macrólidos y su posible beneficio con el tratamiento continuo en niños con sibilancias recurrentes, no existen suficientes pruebas para recomendarlos de forma sistemática y no se trata en esta revisión.

Tabla IV. Tratamiento inicial de mantenimiento en el niño menor de 3 años (modificada de Castillo JA y cols.) $)^{2}$

\begin{tabular}{|c|c|c|c|c|}
\hline \multirow{2}{*}{\multicolumn{2}{|c|}{ Gravedad del asma }} & \multicolumn{2}{|c|}{ Control de base de la enfermedad } & \multirow{3}{*}{$\begin{array}{c}\text { Alivio de } \\
\text { síntomas } \\
\mathrm{AA}-B_{2} \mathrm{AC} \\
\text { a demanda }\end{array}$} \\
\hline & & Elección & Alternativa & \\
\hline \multicolumn{2}{|c|}{ Episódica ocasional } & No precisa & No precisa & \\
\hline \multirow[t]{2}{*}{$\begin{array}{l}\text { Episódica } \\
\text { frecuente }\end{array}$} & IPA- & $\begin{array}{c}\text { Habitualmente } \\
\text { no precisa }\end{array}$ & $\begin{array}{l}\text { Valorar respuesta: } \\
\text { - ARLT } \\
\text { - } \mathrm{GCl} \text { dosis bajas }\end{array}$ & \multirow{2}{*}{$\begin{array}{l}A A-B_{2} A C \\
\text { a demanda }\end{array}$} \\
\hline & $\mathrm{IPA}+$ & $\begin{array}{l}\text { GCl dosis bajas } \\
(\leq 200 \mu \mathrm{g} \mathrm{BD} \mathrm{o} \\
\leq 100 \mu \mathrm{g} \text { Flut })\end{array}$ & ARLT & \\
\hline \multirow{2}{*}{\multicolumn{2}{|c|}{$\begin{array}{c}\text { Persistente moderada } \\
\text { (antes de dar este paso } \\
\text { replantearse el diagnóstico y si } \\
\text { la administración es correcta) }\end{array}$}} & $\begin{array}{l}\mathrm{GCl} \text { dosis medias } \\
(200-400 \mu \mathrm{g} \\
\mathrm{BD} \text { o equiv Flut) }\end{array}$ & $\mathrm{GCl}$ dosis bajas + ARLT & \multirow{2}{*}{$\begin{array}{l}\mathrm{AA}-\mathrm{B}_{2} \mathrm{AC} \\
\text { a demanda }\end{array}$} \\
\hline & & $\begin{array}{r}\text { Valorar resp } \\
\text { Retirar si n } \\
\text { si no exister }\end{array}$ & $\begin{array}{l}\text { ta a los } 3 \text { meses } \\
\text { ay respuesta y } \\
\text { ctores de riesgo }\end{array}$ & \\
\hline \multicolumn{2}{|c|}{ Persistente grave } & \multicolumn{2}{|c|}{$\begin{array}{l}\text { GCl dosis altas (> } 400 \mu \mathrm{g} \text { BD o equiv Flut) } \\
\text { Se puede considerar una o varias: } \\
\text { • Añadir ARLT } \\
\text { - Añadir AA- } \beta_{2} \mathrm{AL} \\
\text { - Añadir GC oral } \\
\end{array}$} & $\begin{array}{l}\mathrm{AA}-\mathrm{B}_{2} \mathrm{AC} \\
\text { a demanda }\end{array}$ \\
\hline \multicolumn{5}{|c|}{$\begin{array}{l}\text { AA- } B_{2} A C: \text { agonista } B_{2} \text { - adrenérgico de acción corta; } A A-B_{2} A L: \text { agonista } B_{2} \text { - adrenérgico de acción larga; } A R L T \text { : } \\
\text { antagonistas de los receptores de los leucotrienos; } B D \text { : budesonida; Flut: fluticasona; GC: glucocorticoide; GCI: } \\
\text { glucocorticoide inhalado; IPA: índice predictivo de asma. }\end{array}$} \\
\hline
\end{tabular}


Corticoides e inhibidores de los leucotrienos. ¿Son igual de efectivos en todos los niños menores de 3 años?, ¿cuándo elegir uno u otro?

\section{Corticoides inhalados}

Las guías y consensos reconocen que los $\mathrm{Cl}$ son el tratamiento de elección en el asma, pero el papel de estos fármacos en la edad preescolar está por determinar debido a la heterogeneidad de fenotipos de sibilantes recurrentes que existen. Aunque se han mostrado seguros, su administración en menores de 3 años debe ser altamente selectiva y estarían indicados, para controlar la enfermedad, en determinados niños con síntomas frecuentes o persistentes (tabla IV)2.

Cuando se compara su eficacia en lactantes y preescolares con sibilancias recurrentes, los resultados sobre la mejoría de los síntomas y función pulmonar son dispares. Es importante distinguir los pacientes con sibilancias recurrentes relacionadas con infecciones respiratorias virales de los de la población de riesgo de sibilancias persistentes o asma.

En lactantes con sibilancias y factores de riesgo de padecer asma, un ensayo clínico aleatorizado (ECA) que comparaba fluticasona frente a placebo durante 12 semanas, mostró una mejoría de los síntomas clínicos y mayor número de días libres de síntomas ${ }^{33}$. Los preescolares con sibilancias e IPA positivo responden a los $\mathrm{Cl}^{34,35}$, pero los efectos son menores que en los escolares. En niños menores de 3 años no hay una clara relación entre la dosis de $\mathrm{Cl}$ y la respuesta clínica. Parece que responden mejor aquellos con síntomas frecuentes, mayores de 2 años y/o con historia familiar de asma ${ }^{36}$.

Una revisión sistemática de ECA con $\mathrm{Cl}$ en preescolares con sibilancias producidas por múltiples desencadenantes (no solo por infecciones víricas), demuestra que los $\mathrm{Cl}$ mejoran el control de los síntomas, la calidad de vida, la función pulmonar y reducen la hiperreactividad bronquial y marcadores de inflamación de la vía aérea ${ }^{37}$. No obstante, la ERS Task Force ${ }^{5}$ sugiere un uso prudente de los $\mathrm{Cl}$ en este tipo de sibilantes. Aconsejan un ensayo con $\mathrm{Cl}$ durante 3 meses y suspender el tratamiento si no se observa mejoría, en vez de subir de escalón terapéutico. Si la respuesta a los $\mathrm{Cl}$ es satisfactoria, estos deben retirarse cuando el niño esté libre de síntomas y reintroducirlos si reaparecen de nuevo.

Algunos autores plantearon la hipótesis de si la introducción precoz y el tratamiento prolongado con $\mathrm{Cl}$ en niños 
seleccionados con sibilancias y elevado riesgo de asma, podría modificar la historia natural del asma y mejorar el pronóstico de esta enfermedad. Ya se había observado que el tratamiento con budesonida inhalada, en pacientes de 5 a 12 años con asma persistente leve 0 moderada, mejoraba el control de la enfermedad mientras recibían el tratamiento, efecto que no persistía al suspenderlo ${ }^{38}$. Resultados similares se han observado al introducir el tratamiento a edades más tempranas. En 285 niños preescolares de 2 a 3 años que habían tenido 4 o más episodios de sibilancias, el tratamiento con fluticasona inhalada (88 $\mu \mathrm{g} / 12$ horas) disminuyó los síntomas de asma y mejoró la función pulmonar respecto al grupo placebo durante los 2 años de tratamiento. Sin embargo, este efecto beneficioso del tratamiento precoz desapareció en el año de seguimiento tras abandonarlo ${ }^{35}$. Estos datos coinciden con otro ECA con fluticasona en donde se evaluó la evolución clínica y funcional en el seguimiento hasta 5 años de edad ${ }^{39,40}$. Se deduce que los $\mathrm{Cl}$ mejoran el control de los síntomas y la calidad de vida de los preescolares con sibilancias recurrentes y riesgo elevado de padecer asma mientras reciben el tratamiento, pero no modifican la historia natural de la enfermedad.
Otra alternativa planteada en niños menores de 3 años con sibilancias y factores de riesgo (hijos de madres asmáticas), fue utilizar $\mathrm{Cl}$ de manera intermitente durante los episodios de sibilancias. En un estudio de 3 años de seguimiento, se observó que la administración de budesonida (400 $\mu \mathrm{g} /$ día) durante 14 días tras el primer episodio, no acortó la duración de los síntomas ni retrasó la aparición de nuevas agudizaciones y evolución a asma respecto al grupo placebo. Por tanto, la aplicación intermitente de $\mathrm{Cl}$ no proporciona ningún beneficio ni a corto ni a largo plazo ${ }^{41,42}$.

Al contrario de lo que ocurre en niños con sibilancias producidas por múltiples desencadenantes o con IPA positivo, no está claro que los $\mathrm{Cl}$ sean eficaces para el control de las sibilancias desencadenados exclusivamente por virus en niños pequeños. Actualmente, no existen evidencias para mantener dosis bajas de $\mathrm{Cl}$ para prevenir estos episodios en lactantes y preescolares $s^{37,43,44}$.

\section{Inhibidores de los leucotrienos}

Los inhibidores de los leucotrienos constituyen una alternativa a los corticoides para iniciar el tratamiento diario en el asma episódica frecuente o pueden asociarse a ellos si no se logra el control de la enfermedad (tabla IV). 
Montelukast es el único fármaco de este grupo terapéutico con el que se han realizado estudios en niños desde los 6 meses y se ha mostrado seguro a partir de esta edad. Tiene un comienzo de acción rápido y se ha descrito que mejora el control del asma, respecto al grupo placebo, en niños de 2-5 años independientemente de si estos estaban o no sensibilizados a alérgenos ${ }^{45}$. También se ha comprobado en preescolares el beneficio de montelukast frente a la hiperrectividad bronquial desencadenada por frío o metacolina.

Son pocos los estudios publicados con montelukast en menores de 3 años, pero hay razones para pensar que pueden ser útiles en estos niños, en los que la mayoría de las sibilancias están asociadas a infecciones víricas de las vías respiratorias, donde se libera gran cantidad de leucotrienos que, además de actuar como potentes broncoconstrictores, favorecen la inflamación neutrofílica en la vía aérea y la hiperreactividad bronquial.

En el estudio PREVIA ${ }^{46}$, en el que se siguieron durante 12 meses más de 500 niños de 2-5 años con asma leve intermitente que recibieron montelukast o placebo, se vio un claro empeoramiento clínico en épocas en las que predominan las infecciones víricas (otoño/invierno), pero el número de exacerbaciones y visitas médicas fue menor en el grupo que recibió montelukast.

Dado que existe un predominio estacional en las infecciones víricas y que estas son un desencadenante importante de exacerbaciones de sibilancias/asma en la infancia, algunos autores han puesto de manifiesto las ventajas de iniciar o añadir montelukast al tratamiento antiasmático habitual a partir de septiembre, antes de comenzar la epidemia. Se ha observado que el mayor beneficio de esta pauta se obtenía en los varones menores de 5 años $^{47}$. Otra posible futura hipótesis de tratamiento sería administrar montelukast sólo en las agudizaciones, pero actualmente no existe suficiente evidencia para hacer estas recomendaciones. Robertson et al. ${ }^{48}$ han observado que instruir a los padres en la administración de un ciclo corto de montelukast, al menos 7 días, al inicio de los episodios agudos en niños con asma intermitente, disminuye las visitas no programadas por asma, la ausencia escolar y la pérdida de días de trabajo de los padres en los 12 meses de seguimiento.

Independientemente de la respuesta de montelukast frente al asma/sibilancias desencadenadas por infecciones víricas, también se han publicado dos 
ECA que comparan montelukast con placebo en niños seleccionados con factores de riesgo de atopia menores de 26 meses $^{49}$ y de 2-5 años ${ }^{50}$. En ambos, aunque el número de casos es pequeño, se observó una mejoría significativa de la función pulmonar y disminución del óxido nítrico (marcador de inflamación eosinofílica) exhalado tras 4 semanas de tratamiento con montelukast respecto al grupo placebo.

Pocos estudios evalúan el efecto controlador de los $\mathrm{Cl}$ frente a montelukast en niños. Cuando se comparan $\mathrm{Cl}$ y montelukast en el tratamiento del asma persistente leve a moderada, los resultados de un metaanálisis que incluyen niños mayores de 2 años y adultos, muestran superioridad de los corticoides en el control de los síntomas y la función pulmonar $^{51}$. No existen ensayos clínicos comparativos en menores de 2 años.

Recientemente se ha publicado el primer ECA que compara la eficacia a largo plazo de montelukast y budesonida, que incluye niños preescolares ${ }^{52}$. Se ha realizado durante un año en 395 pacientes de 2-8 años con sibilancias recurrentes o asma leve. Los autores concluyen que ambos fármacos son bien tolerados $y$, aunque parece que la respuesta es más satisfactoria con los $\mathrm{Cl}$ durante los primeros meses, no encuen- tran diferencias en el control de la enfermedad a los 12 meses de tratamiento. El trabajo tiene varias debilidades metodológicas en diseño y análisis de los resultados para tomar decisiones, entre otras que no es ciego y no separa grupos por factores de riesgo de asma ni por edad, a pesar de que $2 / 3$ de los pacientes tienen menos de 5 años, edad en la que existe mayor heterogeneidad en la respuesta al tratamiento que en los niños mayores.

La mayoría de estudios con montelukast se han realizado en niños con asma intermitente o persistente leve, no existe evidencia para recomendarlos como monoterapia en el asma de mayor gravedad. También pueden ser útiles para disminuir las exacerbaciones desencadenadas por virus. La gran ventaja de este fármaco es su administración por vía oral y su cómoda posología. No obstante, al igual que ocurre con los corticoides, no tenemos suficientes herramientas para afirmar con seguridad qué niños van a responder al tratamiento.

\section{En el tratamiento farmacológico de mantenimiento debemos recordar}

- Los fármacos recomendados en los niños menores de 3 años son los corticoides inhalados y los inhibidores de los leucotrienos. En estas 
edades se ha observado que unos pacientes responden mejor a uno, otros a otro y algunos no responden a ninguno de estos fármacos.

- Los corticoides inhalados son el tratamiento de elección en el asma. En preescolares, la respuesta es más satisfactoria en niños con antecedentes de atopia.

- No existen evidencias para recomendar dosis bajas de corticoides inhalados en la prevención de episodios de sibilancias desencadenadas por virus en lactantes y preescolares.

- La aplicación intermitente de corticoides inhalados durante los episodios de sibilancias, incluso en pacientes de alto riesgo, no proporciona ningún beneficio a corto ni a largo plazo.

- Los inhibidores de los leucotrienos constituyen una alternativa a los corticoides inhalados en niños con asma leve y podrían utilizarse para disminuir las exacerbaciones inducidas por virus en estos casos. Ante mayor gravedad de la enfermedad y sobre todo en niños atópicos, se recomienda iniciar el tratamiento con corticoides inhalados.

- Montelukast podría utilizarse asociado a corticoides inhalados si no existe control de los síntomas o en las estaciones epidémicas de infecciones víricas.

\section{Bibliografía}

1. Warner JO, Naspitz CK. Third International Pediatric Consensus statement on the management of childhood asthma. International Pediatric Asthma Consensus Group. Pediatr Pulmonol. 1998;25:1-17.

2. Castillo JA, de Benito J, Escribano A y col. Consenso sobre tratamiento del asma en pediatría. An Pediatr (Barc). 2007;67:253-73.

3. Bacharier LB, Boner A, Carlsen KH. Diagnosis and treatment of asthma in childhood: a PRACTALL consensus report. Allergy. 2008;63: 5-34.

4. Praena Crespo M. ¿A qué llamamos asma infantil? Rev Pediatr Aten Primaria. 2005;7 (2 Sup):S13-27.

5. Brand PLP, Baraldi E, Bisgaard H. Definition, assessment and treatment of wheezing disorders in preschool children: an evidence-based approach. Eur Respir J. 2008;32:1096-110.

6. Phelan PD, Robertson CF, Olinsky A. The Melbourne Asthma Study: 1964-1999. J Allergy Clin Immunol. 2002;109:189-94.

7. Taussig LM, Wright AL, Holberg CJ, Halonen $M$, Morgan WJ, Martínez FD. Tucson Children's Respiratory Study: 1980 to present. J Allergy Clin Immunol. 2003;111:661-75.

8. Martinez FD, Godfrey S. Management of wheezing in infants and preschool children. En: 
Martinez FD, Godfrey S, edit. Wheezing disorders in the preschool child. New York: Martin Dunitz; 2003. p. 123-42.

9. Boehmer AL, Merkus PJ. Asthma therapy for children under 5 years of age. Curr Opin Pulm Med. 2006;12:34-41.

10. Saglani S, Malmström K, Pelkonen AS. Airway remodeling and inflammation in symptomatic infants with reversible airflow obstruction. Am J Respir Crit Care Med. 2005;171:722-7.

11. Saglani S, Payne DN, Zhu J. Early detection of airway wall remodeling and eosinophilic inflammation in preschool wheezers. Am J Respir Crit Care Med. 2007;176:858-64.

12. Castro-Rodríguez JA, Holberg CJ, Wright AL, Martinez FD. A clinical index to define risk of asthma in young children with recurrent wheezing. Am J Respir Crit Care Med. 2000;162: 1403-6.

13. Guilbert TW, Morgan WJ, Zeiger RS. Atopic characteristics of children with recurrent wheezing at high risk for the development of childhood asthma. J Allergy Clin Immunol. 2004;14:1282-7.

14. Korta J, Valverde J, Praena M. La educación terapéutica en el asma. An Pediatr (Barc). 2007;66:496-517.

15. Gdalevich $M$, Mimouni $D$, Mimouni $M$. Breast-feeding and the risk of bronchial asthma in childhood: a systematic review with metaanalysis of prospective studies. J Pediatr. 2001; 139:261-6.

16. Zutavern A, Brockow I, Schaaf $B$, von Berg $A$, Diez $U$, Borte $M$, et al. Timing of solid introduction in relation to eczema, asthma, allergic rhinitis, and food and inhalants sensitization at the age of 6 years: results from the prospective birth cohort study LISA. Pediatrics. 2008;121: e44-52.

17. Cates CJ, Jefferson TO, Rowe BH. Vacci- nes for preventing influenza in people with asthma. Cochrane Database of Systematic Reviews 2008, Issue 2. Art. No.: CD000364.

18. Lannerö E, Wickman M, Pershagen, Nordvall L. Maternal smoking during pregnancy increases the risk of recurrent wheezing during the first years of life (BAMSE). Respir Res. 2006;7:3.

19. Buñuel Álvarez JC, Montón Álvarez JL. Los hijos de madres que fumaron durante la gestación presentaron una mayor incidencia de enfermedades respiratorias de vías bajas durante los dos primeros años de vida. Evid Pediatr. 2006;2: 30 [consultado el 13/01/2009]. Disponible en www.aepap.org/EvidPediatr/index.htm

20. Hofhuis W, de Jongste JC, Merkus PJ. Adverse health effects of prenatal and postnatal tobacco smoke exposure on children. Arch Dis Child. 2003;88:1086-90.

21. Piippo-Savolainen E, Korppi M. Wheezy babies-wheezy adults? Review on long-term outcome until adulthood after early childhood wheezing. Acta Paediatr. 2008;97:5-11.

22. Úbeda Sansano MI. Tratamiento de la crisis asmática en atención primaria. Acta Pediatr Esp. 2004;62:153-62.

23. Smith SR, Baty JD, Hodge D III. Validation of the pulmonary score: an asthma severity score for children. Acad Emerg Med. 2002;9:99-104.

24. Chavasse R, Seddon P, Bara A, McKean $M$. Short acting beta2-agonists for recurrent wheeze in children under two years of age. Cochrane Database of Systematic Reviews 2002, Issue 2. Art. No.: CD002873.

25. Cates CJ, Crilly JA, Rowe BH. Cámaras espaciadoras versus nebulizadores para el tratamiento del asma aguda con betaagonistas (Revisión Cochrane traducida). En: La Biblioteca Cochrane Plus, 2008 Número 4. Oxford: Update Software Ltd. Disponible en: http://www.upda te-software.com. (Traducida de The Cochrane 
Library, 2008 Issue 3. Chichester, UK: John Wiley \& Sons, Ltd.).

26. Benito Fernández J, Trebolazábala Quirante N, Landa Garriz M, Mintegui Raso S, González Díaz C. Broncodilatadores inhalados mediante MDI con cámara espaciadora en urgencias pediátricas: ¿cuál es la dosis? An Pediatr (Barc). 2006;64:46-51.

27. Everard $M$, Bara $A$, Kurian $M, N^{\prime}$ Diaye $T$, Ducharme $F$, Mayowe $V$. Anticholinergic drugs for wheeze in children under the age of two years. Cochrane Database of Systematic Reviews 2005, Issue 3. Art. No.: CD001279.

28. Vuillermin $P$, South $M$, Robertson $C$. Parent-initiated oral corticosteroid therapy for intermittent wheezing illnesses in children (Cochrane Review). En: The Cochrane Library, Issue 3. Chichester, UK: John Wiley \& Sons Ltd; 2006.

29. Ducharme FM, Chabot G, Polychronakos C, Glorieux F, Mazer B. Safety profile of frecuent short courses of oral glucocorticoids in acute pediatric asthma: impact on bone metabolism, bone density and adrenal function. Pediatrics. 2003;111:376-83.

30. Jiménez Cortés A, Praena Crespo M, Lora Espinosa A, Grupo de Vías Respiratorias de la AEPap. Normas de calidad para el tratamiento de la crisis de asma en el niño y adolescente. Documentos técnicos del GVR (publicación DT-GVR1) [consultado el 10/10/2008]. Disponible en www.aepap.org/gvr/protocolos.htm

31. Jiménez Cortés A, Mola Caballero de Rodas P. Tratamiento de la crisis de asma. En: Cano A, Díaz-Vazquez C, Montón JL, editores. Asma en el niño y Adolescente. 2. ${ }^{a}$ Edición. Madrid: Ergon; 2004. p.115-26.

32. Pérez-Yarza EG, Sardón Prado O, Korta Murua J. Sibilancias recurrentes en menores de tres años: evidencias y oportunidades. An Pediatr (Barc). 2008;69:369-82.
33. Chavasse RJ, Bastian-Lee $\mathrm{Y}$, Richter $\mathrm{H}$, Hilliard T, Seddon P. Persistent wheezing in infants with an atopic tendency responds to inhaled fluticasone. Arch Dis Child. 2001;85:143-8.

34. Teper AM, Kofman CD, Szulman GA, Vidaurreta SM, Maffey AF. Fluticasone improves pulmonary function in children under 2 years old with risk factors for asthma. Am J Respir Crit Care Med. 2005;171:587-90.

35. Guilbert TW, Morgan WJ, Zeiger RS. Long-term inhaled corticosteroids in preschool children at high risk for asthma. N Engl J Med. 2006;354:1985-97.

36. Roorda RJ, Mezei G, Bisgaard H, Maden C. Response of preschool children with asthma symptoms to fluticasone propionate. J Allergy Clin Immunol. 2001;108:540-6.

37. Kaditis AG, Winnie $G$, Syrogiannopoulos GA. Antiinflammatory pharmacotherapy for wheezing in preschool children. Pediatr Pulmonol. 2007;42:407-20.

38. The Childhood Asthma Management Program Research Group. Long-term effects of budesonide or nedocromil in children with asthma. N Engl J Med. 2000;343:1054-63.

39. Murray CS, Woodcock A, Langley SJ, Morris J, Custovic A. Secondary prevention of asthma by the use of inhaled fluticasone wheezy infants (IFWIN): double-blind, randomised, controlled study. Lancet. 2006;368:754-62.

40. García Vera C, Ibáñez Pradas V. El uso de propionato de fluticasona inhalado no parece modificar la historia natural del asma en niños menores de cinco años con sibilancias. Evid Pediatr. 2006;2:77 [consultado el 13/01/2009]. Disponible en www.aepap.org/EvidPediatr/index.htm

41. Bisgaard $H$, Hermansen $M N$, Loland $L$, Halkjaer LB, Bulchvald F. Intermitent inhaled cortocosteroids in infants with episodic wheezing. N Engl J Med. 2006;354:1998-2005. 
42. Fino E, González de Dios J. En niños menores de tres años con alto riesgo de asma y episodios de sibilancias, la administración precoz de corticoides inhalados de forma intermitente no produce beneficios en la evolución del espasmo bronquial de episódico a persistente ni tampoco efectos a corto plazo. Evid Pediatr. 2006;2:42 [consultado el 13/01/2009]. Disponible en www. aepap.org/EvidPediatr/index.htm

43. McKean M, Ducharme F. Inhaled steroids for episodic viral wheeze of childhood. Cochrane Database of Systematic Reviews 2000, Issue 1. Art. No.: CD001107.

44. Schokker S, Kooi EM, de Vries TW, Brand PL, Mulder PG, Duiverman EJ, et al. Inhaled corticosteroids for recurrent respiratory symptoms in preschool children in general practice: randomized controlled trial. Pulm Pharmacol Ther. 2008;21:88-97.

45. Knorr B, Franchi LM, Bisgaard H. Montelukast, a leukotriene receptor antagonist, for the treatment of persistent asthma in children aged 2 to 5 years. Pediatrics. 2001;108:e48.

46. Bisgaard H, Zielen S, Garcia-Garcia ML. Montelukast reduces asthma exacerbations in 2to 5-year-old children with intermittent asthma. Am J Respir Crit Care Med. 2005;171:315-22.

47. Johnston NW, Mandhane PJ, Dai J. Attenuation of the September epidemic of asthma exacerbations in children: a randomized, contro- lled trial of montelukast added to usual therapy. Pediatrics. 2007;120:e702-712.

48. Robertson CF, Price D, Henry R. Shortcourse montelukast for intermittent asthma in children: a randomized controlled trial. Am J Respir Crit Care Med. 2007:175:323-9.

49. Straub DA, Moeller A, Minocchieri S. The effect of montelukast on lung function and exhaled nitric oxide in infants with early childhood asthma. Eur Respir J. 2005;25:289-94.

50. Straub DA, Minocchieri S, Moeller A, Hamacher J, Wildhaber JH. The effect of montelukast on exhaled nitric oxide and lung function in asthmatic children 2 to 5 years old. Chest. 2005; 127:509-14.

51. Ng D, Di Salvio F, Hicks G. Agentes antileucotrienos comparados con corticosteroides inhalados para el tratamiento del asma recurrente y/o crónica en adultos y niños (Revisión Cochrane traducida). En: La Biblioteca Cochrane Plus, 2008 Número 4. Oxford: Update Software Ltd. Disponible en: http://www.update-software. com. (Traducida de The Cochrane Library, 2008 Issue 3. Chichester, UK: John Wiley \& Sons, Ltd.).

52. Szefler SJ, Baker JW, Uryniak T, Goldman $M$, Silkoff PE. Comparative study of budesonide inhalation suspension and montelukast in young children with mild persistent asthma. J Allergy Clin Immunol. 2007;120:1043-50. 